\title{
Pelvic schwannoma in the right parametrium
}

This article was published in the following Dove Press journal:

International Journal of General Medicine

7 March 2013

Number of times this article has been viewed

\section{Nikolaos Machairiotis ${ }^{4}$ \\ Paul Zarogoulidis ${ }^{3}$ \\ Aikaterini Stylianaki' \\ Eleni Karatrasoglou ${ }^{4}$ \\ Georgia Sotiropoulou ${ }^{4}$ \\ Alvin Floreskou ${ }^{4}$ \\ Eleana Chatzi ${ }^{4}$ \\ Athanasia Karamani ${ }^{4}$ \\ Georgia Liapi ${ }^{5}$ \\ Eleni Papakonstantinou ${ }^{5}$ \\ Nikolaos Katsikogiannis ${ }^{\prime}$ \\ Nikolaos Courcoutsakis ${ }^{2}$ \\ Christodoulos Machairiotis ${ }^{4}$ \\ 'Surgery Department, ${ }^{2}$ Radiology \\ Department, University General \\ Hospital of Alexandroupolis, \\ Democritus University of Thrace, \\ Alexandroupolis, Greece; ${ }^{3}$ Pulmonary \\ Department, G Papanikolaou General \\ Hospital, Aristotle University \\ of Thessaloniki, Thessaloniki, Greece; \\ ${ }^{4}$ Obstetric Gynecology Department, \\ ${ }^{5}$ Pathology Department, Thriasio \\ General Hospital, Athens, Greece}

Correspondence: Paul Zarogoulidis Pulmonary Department, G Papanikolaou General Hospital, Aristotle University of Thessaloniki, Thessaloniki, Greece

Tel +30 697727 I974

$\mathrm{Fax}+302310992433$

Email pzarog@hotmail.com
Abstract: Neurilemomas are benign usually encapsulated nerve sheath tumors derived from the Schwann cells. These tumors commonly arise from the cranial nerves as acoustic neurinomas but they are extremely rare in the pelvis and the retroperitoneal area (less than $0.5 \%$ of reported cases), unless they are combined with von Recklinghausen disease (type 1 neurofibromatosis). We report the case of a 58-year-old female with pelvic schwannoma, $6.5 \times 5.5 \mathrm{~cm}$ in size, in the right parametrium. This is the first case reported in the literature. Based on the rarity of this tumor and in order to ensure optimum treatment and survival for our patient, we performed laparotomy with total abdominal hysterectomy and en-block tumor excision. A frozen section was taken during the surgery before complete resection of the mass, which was ambiguous. Because of the possibility of malignancy, complete excision of the mass was performed, with pelvic blunt dissection. Histological examination showed a benign neoplasm, originating from the cells of peripheral nerve sheaths; diagnosis was a schwannoma. There were degenerative areas, including cystic degeneration, hemorrhagic infiltrations, ischemic foci with pycnotic cells, and collagen replacement. Pelvic schwannomas are rare neoplasms that can be misdiagnosed. Laparoscopy is a safe and efficient option for approaching benign pelvic tumors and might offer the advantage of better visualization of structures due to the magnification in laparoscopic view, especially in narrow anatomic spaces.

Keywords: pelvis, schwannoma, laparoscopy, neoplasm

Neurilemomas are benign usually encapsulated nerve sheath tumors derived from the Schwann cells. They constitute one of the most common types of benign peripheral nerve sheath tumors. These tumors may be common in cranial and peripheral nerves but they are rarely located in the pelvis. ${ }^{1,2}$ Schwannomas can occur sporadically or as manifestations of genetic conditions, such as neurofibromatosis 1 and 2. Pelvic schwannomas have no specific radiologic features and are often considered to be urologic diseases or gynecologic masses., ${ }^{3,4}$ We report the case of a female with a pelvic schwannoma in the right parametrium.

\section{Case presentation}

A 58-year-old Caucasian woman was admitted to the gynecology out-patient department for management of increasing abdominal distension and sustained pain in the right bottom quadrant of her abdomen that had been ongoing for 3 months, was independent of activities, and was without any signs of neurovascular deficit. She also mentioned a 1-week history of vomiting and nausea. Around that time she also started to suffer from increased frequency of minor urinary incontinence. In the last 48 hours the 
pain was located in the right iliac fossa. Her medical history and family history were unremarkable. Her surgical history included only an appendectomy as a child, while her obstetric history was remarkable only for five natural childbirths. A bimanual pelvic exam revealed an agile cervix and a large, solid, ovoid, palpable mass in the right parametrium, which was particularly sensitive to touch. Her routine hematological investigation and urine analysis as well as examination of several tumor markers, including carcinoembryonic antigen, $\alpha$-fetoprotein, and carbohydrate antigen 19.9, were all within normal limits. Initial radiographs failed to reveal tumor-like masses. A gynecologic ultrasonography was performed, which revealed an increase in the size of the right ovary $(56.6 \times 35 \mathrm{~mm})$ and the possible presence of a cystic formation measuring $32.5 \mathrm{~mm}$. Computer tomography confirmed the presence of a mass in the anatomic region of the right ovary, corresponding to the ovary's schwannoma (Figure 1).

The preoperative findings showed we had to deal with a retroperitoneal tumor of unknown pathology in a menopausal woman. In order to ensure the optimum treatment and survival for our patient, we performed laparotomy with total abdominal hysterectomy and en-block tumor excision. The abdomen was opened through a lower midline incision. A large retroperitoneal mass, measuring $6.5 \times 5.5 \mathrm{~cm}$, was noted in the right parametrium and was in close proximity to the interal-iliac vein, which was ligated (Figure 2). A frozen section was taken during the surgery before complete resection of the mass, which was ambiguous. Because of the possibility of malignancy, complete excision of the mass was performed with pelvic

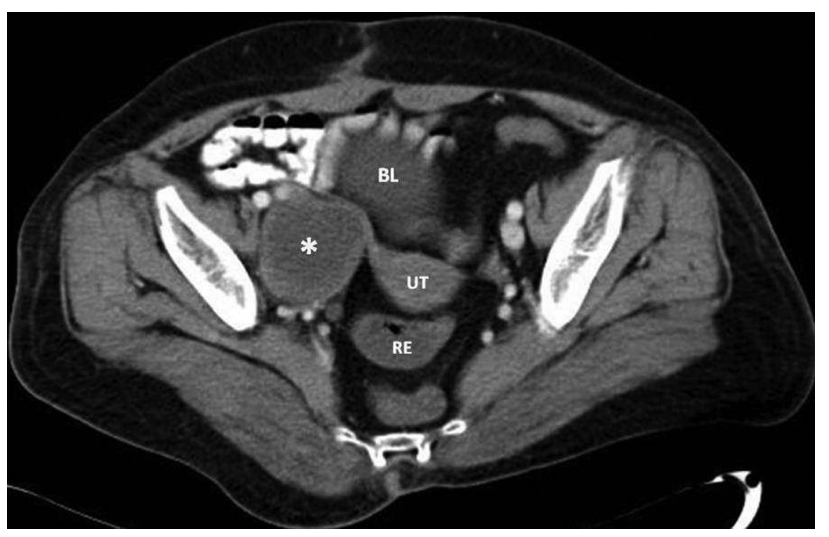

Figure I Axial, enhanced computed tomography image of the pelvis demonstrates a mass in the anatomic region of the right ovary, corresponding to the ovary's schwannoma.

Note: The enlarged ovary is well delineated, with internal low density and enhanced peripherally.

Abbreviations: UT, uterus; BL, urinary bladder; RE, rectum.

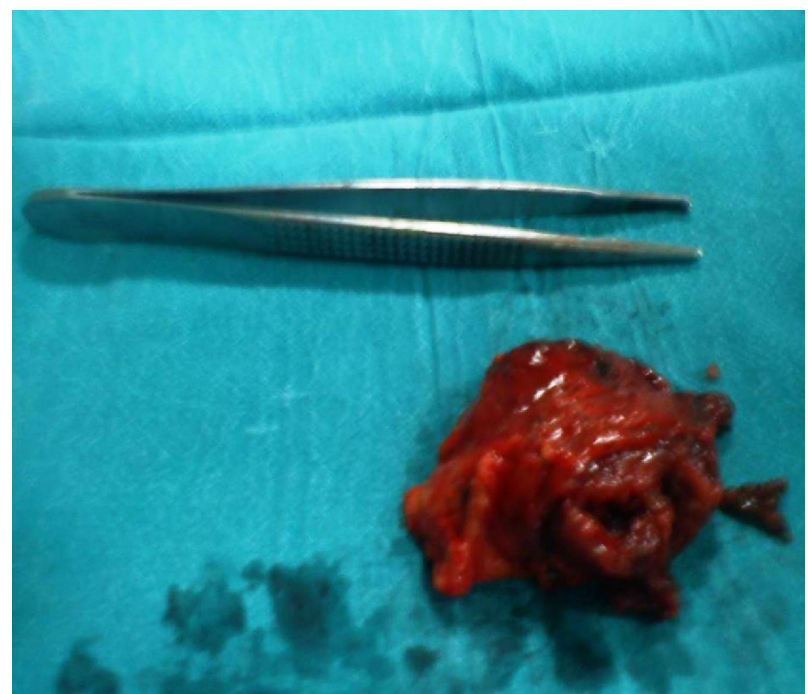

Figure 2 Surgically resected lesion, which was noted in the right parametrium measuring $6.5 \times 5.5 \mathrm{~cm}$.

blunt dissection. Histological examination showed a benign neoplasm originating from the cells of the peripheral nerve sheath; diagnosis was a schwannoma. The tumor showed a biphasic pattern consisting mainly of cellular areas with nuclear palisading (Antoni A) and few hypocellular areas with loose texture (Antoni B) (Figure A, B and C). The tumor cells were spindle-shaped with spindle nuclei without atypia.

Degenerative areas within the tumor were seen, including cystic degeneration, hemorrhagic infiltrations and ischemic foci with pycnotic cells due to vessel infarction and fibrinoid necrosis. Immunohistochemical studies were performed with a panel of antibodies including S-100 protein,

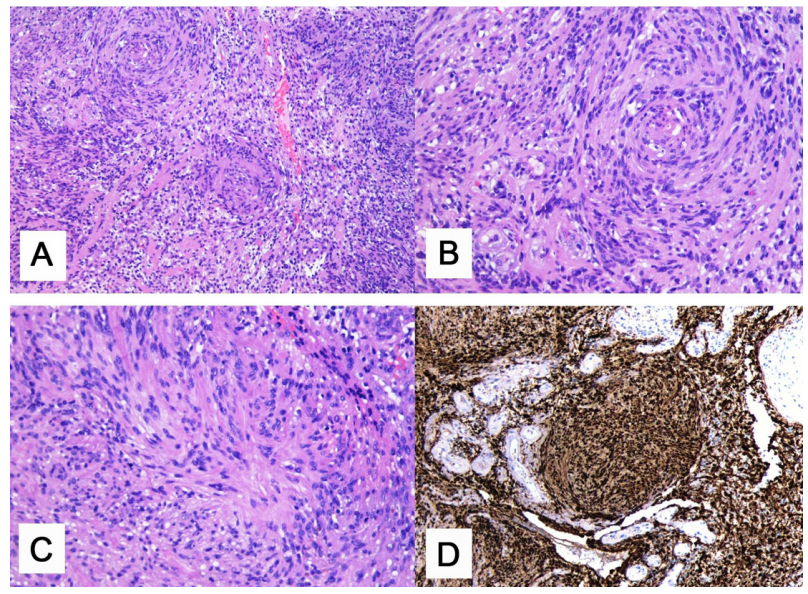

Figure 3 Histological findings. (A) Biphasic pattern mainly with Antoni A and few hypocellular, Antoni B, areas (x100). (B-C) Higher magnification of Antoni A areas $(x 200)$. (D) S- 100 protein positivity of the elongated tumor cells $(x 200)$. 
smooth muscle actin (SMA), and CD68. The tumor cells were strongly positive against S-100 protein (Figure D). SMA staining was negative and CD68 revealed numerous histiocytes in the interstitial stroma. Postoperatively, the patient showed a good recovery and was discharged on postoperative day 6 .

\section{Conclusion}

Schwannomas are nerve sheath tumors that arise in peripheral, cranial, or visceral nerves at any anatomic site of the human body. ${ }^{5,6}$ These tumors commonly arise from the cranial nerves as acoustic neurinomas but they are extremely rare in the pelvis and the retroperitoneal area (less than $0.5 \%$ of reported cases), unless they are combined with von Recklinghousen disease (type 1 neurofibromatosis). ${ }^{6}$ Neurilemomas are nonaggressive, slow-growing, solitary neoplasms with an extremely low possibility of malignant transformation or recurrence after excision. ${ }^{7}$ Macroscopically schwannomas are solitary, well-circumscribed, encapsulated tumors. ${ }^{4}$ Histologically, neurilemomas are characterized by the presence of Antoni A and Antoni B bodies. Antoni A bodies represent a disposition in a Verocay body, and Antoni B tissue is a loose hypocellular myxoid region of microcysts. ${ }^{8,9}$ Immunohistochemistry is positive for S-100, vimentin, and neuron-specific enolase but negative for SMA and CD117. ${ }^{7}$

As a result of their slow growth rate and anatomic location, pelvic schwannomas remain asymptomatic and are either incidentally discovered during a medical investigation for unrelated symptoms or are discovered as soon as they are sufficiently large to cause a mass effect. ${ }^{7,9}$ This mass effect can lead to pain in the pelvic area and lower back and a sense of heaviness accompanied with urinary and digestive symptoms caused by bladder and bowel compression. ${ }^{9}$ Pelvic neurilemomas are not easily diagnosed. The clinical signs and symptoms are not pathognomonic for this pathologic situation.

On the occasion of our case report, we conducted a literature review and documented the cases of pelvic schwannomas of gynecologic interest. The small number of these cases indicates the rarity of retroperitoneal pelvic schwannomas. One case was a retroperitoneal pelvic schwannoma located in the right paracolpium; ${ }^{10}$ another case was a schwannoma present at the L5 vertebral body and causing pelvic pain. ${ }^{11}$ Takeuchi et al reported eleven presacral and lateral pelvic region neurilemomas, eight of which were located in the right or left presacral area and three in the lateral extraperitoneal region. ${ }^{12}$ One pelvic schwannoma of the anterior surface of the sacrum mimicking an ovarian cyst was also reported. ${ }^{13}$ An additional case of a retroperitoneal schwannoma localized in the pelvic cavity with complete cystic degeneration, mimicking an ovarian carcinoma, was reported ${ }^{14}$ as well as one presacral neurilemoma. ${ }^{15}$ One pelvic retroperitoneal neurilemoma arose in the fallopian tube, ${ }^{16}$ and one malignant schwannoma arose in the cervix. ${ }^{17}$ Yadav et al reported one case of neurilemoma located in the right adnexum, ${ }^{18}$ and Sinha et al reported two cases of pelvic schwannomas located in the broad ligament, with the clinical expression of a broad ligament myoma. ${ }^{9}$ One reported case of neurilemoma of the pelvis mimicked a myoma of the uterus. ${ }^{19}$ Pelvic tumors and, more specifically, pelvic schwannomas can cause chronic pelvic pain, as was reported in a case of a femoral nerve schwannoma that was clinically expressed with the symptom of chronic pelvic pain. ${ }^{6}$ There were two cases of obturator nerve tumors arising from the Schwann cells, one expressed as a pelvic tumor and the other as an ovarian tumor. ${ }^{20}$ Nine cases of schwannomas mimicking ovarian malignancies have also been reported. ${ }^{21,22}$

Pelvic schwannomas, as mentioned above, are easily misdiagnosed due to the lack of specific symptoms. Their therapy is considered to be complete excision of the tumor either laparoscopically or with open abdominal surgery. ${ }^{23}$ Considering that the vast majority of schwannomas are benign tumors, simple tumor enucleation could also be effective. ${ }^{7}$ In these surgical approaches it is important that the surgeon not cause neural lesions in the patient after surgery. ${ }^{7}$ The presacral-retrorectal space is considered to be a downward extension of the retroperitoneal space because it communicates superiorly with the latter at the level of peritoneal reflection (S2-3 vertebrae). ${ }^{23,24}$ Surgical extirpation of presacral and pelvic tumors may have several operative difficulties due to limited access and poor visualization in a narrow pelvis. ${ }^{23,24}$

Schwannomas are solitary, well-circumscribed, encapsulated tumors and do not invade local tissues. ${ }^{4}$ Due to these characteristics they are easily dissected from adjacent tissues, which makes laparoscopic resection possible. Laparoscopy might also facilitate dissection due to magnification of the anatomic elements in the narrow pelvis. ${ }^{23}$ The literature review indicated that laparoscopic excision of these neural tumors is the therapy of choice. Of note is that many pelvic schwannomas were misdiagnosed and were discovered during an operation that was considered to be the optimal therapy for the initial diagnosis.

Laparoscopy is a safe and efficient option for approaching benign pelvic tumors and might offer the advantage of better 
visualization of structures due to the magnification of the laparoscopic view, especially in narrow anatomic spaces. ${ }^{23}$

\section{Acknowledgments}

$\mathrm{N}$ Machairiotis, P Zarogoulidis, and A Stylianaki made substantial contributions to conception and design and writing of the manuscript; G Sotiropoulou, E Chatzi, A Karamani, G Liapi, and E Papakonstantinou made substantial contributions to acquisition of data and analysis and interpretation of pathological data; N Courcoutsakis made substantial contributions to analysis and interpretation of radiological data; Elena Karatrasoglou, Nikolaos Katsikogiannis and Alvin Floreskou assisted in the editing of the manuscript. C Machairiotis gave final approval of the version to be published. Written consent for this case study was provided by the patient.

\section{Disclosure}

The authors report no conflicts of interest in this work.

\section{References}

1. Rao W, Wang G, Xiu D. Laparoscopic resection of a retroperitoneal schwannoma adherent to vital vessels. Surg Laparosc Endosc Percutan Tech. 2009;19(1):e21-e23.

2. Funamizu N, Sasaki A, Matsumoto T, Inomata M, Shiraishi N, Kitano S. Laparoscopic resection of a retroperitoneal schwannoma behind the lesser omental sac. Surg Laparosc Endosc Percutan Tech. 2004;14(3):175-177.

3. Mehta M, Thurston WA, Merchant N, Murphy KJ. Obturator nerve schwannoma presenting as an adnexal mass: case report. Can Assoc Radiol J. 1999;50(1):20-22.

4. Daneshmand S, Youssefzadeh D, Chamie K, et al. Benign retroperitoneal schwannoma: a case series and review of the literature. Urology. 2003;62(6):993-997.

5. Borghese M, Corigliano N, Gabriele R, et al. Benign schwannoma of the pelvic retroperitoneum. Report of a case and review of the literature. G Chir. 2000;21(5):232-238.

6. Dawley B. A retroperitoneal femoral nerve schwannoma as a cause of chronic pelvic pain. J Minim Invasive Gynecol. 2008;15(4):491-493.

7. Strauss DC, Qureshi YA, Hayes AJ, Thomas JM. Management of benign retroperitoneal schwannomas: a single-center experience. Am J Surg. 2011;202(2):194-198.
8. White W, Shiu MH, Rosenblum MK, Erlandson RA, Woodruff JM. Cellular schwannoma. A clinicopathologic study of 57 patients and 58 tumors. Cancer. 1990;66(6):1266-1275.

9. Sinha R, Sundaram M, Hegde A, Mahajan C. Pelvic schwannoma masquerading as broad ligament myoma. J Minim Invasive Gynecol. 2008;15(2):217-219.

10. Ueda M, Okamoto Y, Ueki M. A pelvic retroperitoneal schwannoma arising in the right paracolpium. Gynecol Oncol. 1996;60(3): 480-483.

11. Shoher A, Arbab F, Lucci A Jr. Giant pelvic schwannoma with ancient change. J Am Coll Surg. 2003;197(1):163.

12. Takeuchi M, Matsuzaki K, Nishitani H, Uehara H. Ancient schwannoma of the female pelvis. Abdom Imaging. 2008;33(2):247-252.

13. Ibraheim M, Ikomi A, Khan F. A pelvic retroperitoneal schwannoma mimicking an ovarian dermoid cyst in pregnancy. J Obstet Gynaecol. 2005;25(6):620-621.

14. Aran T, Guven S, Gocer S, Ersoz S, Bozkaya H. Large retroperitoneal schwannoma mimicking ovarian carcinoma: case report and literature review. Eur J Gynaecol Oncol. 2009;30(4):446-448.

15. Song JY, Kim SY, Park EG, et al. Schwannoma in the retroperitoneum. J Obstet Gynaecol Res. 2007;33(3):371-375.

16. Duran B, Guvenal T, Yildiz E, Cetin M, Erden O, Demirkoprulu N. An unusual cause of adnexal mass: fallopian tube schwannoma. Gynecol Oncol. 2004;92(1):343-346.

17. Lallas TA, Mehaffey PC, Lager DJ, Van Voorhis BJ, Sorosky JI. Malignant cervical schwannoma: An unusual pelvic tumor. Gynecol Oncol. 1999;72(2):238-242.

18. Yadav Y, Onon T, Sukumar S. Conservative management of a pelvic Schwannoma presenting as an adnexal mass. J Obstet Gynaecol. 2008;28(3):364-365.

19. Chen CH, Jeng CJ, Liu WM, Shen J. Retroperitoneal schwannoma mimicking uterine myoma. Taiwan J Obstet Gynecol. 2009;48(2): 176-177.

20. Ningshu L, Min Y, Xieqiao Y, Yuanqing Y, Xiaoqiang M, Rubing L. Laparoscopic management of obturator nerve schwannomas: experiences with 6 cases and review of the literature. Surg Laparosc Endosc Percutan Tech. 2012;22(2):143-147.

21. Ozat M, Altinkaya SO, Gungor T, et al. Extraovarian conditions mimicking ovarian cancer: a single center experience of 15 years. Arch Gynecol Obstet. 2011;284(3):713-719.

22. Korkontzelos I, Tsimoyiannis E, Zagaliki A, Demou A, Karabina E, Antoniou N. Pelvic retroperitoneal schwannoma presenting as a gynecologic mass: case report. Eur J Gynaecol Oncol. 2005;26(1):117-119.

23. Konstantinidis K, Theodoropoulos GE, Sambalis G, et al. Laparoscopic resection of presacral schwannomas. Surg Laparosc Endosc Percutan Tech. 2005;15(5):302-304.

24. Wolpert A, Beer-Gabel M, Lifschitz O, Zbar AP. The management of presacral masses in the adult. Tech Coloproctol. 2002;6(1):43-49.
International Journal of General Medicine

\section{Publish your work in this journal}

The International Journal of General Medicine is an international, peer-reviewed open-access journal that focuses on general and internal medicine, pathogenesis, epidemiology, diagnosis, monitoring and treatment protocols. The journal is characterized by the rapid reporting of reviews, original research and clinical studies across all disease areas.

\section{Dovepress}

A key focus is the elucidation of disease processes and management protocols resulting in improved outcomes for the patient.The manuscript management system is completely online and includes a very quick and fair peer-review system. Visit http://www.dovepress.com/ testimonials.php to read real quotes from published authors. 